\title{
Association of potato and other starchy vegetable consumption with cardiovascular diseases risk factors in Iranian elderly men
}

Mursal Basiry

Ghazanfer Institute of health sciences https://orcid.org/0000-0003-1380-1870

Elnaz Daneshzad

Tehran University of Medical Sciences

Hadis Mozaffari

Tehran University of Medical Sciences

Leila Azadbakht ( $\square$ azadbakhtleila@gmail.com )

Tehran University of Medical Sciences

\section{Research}

Keywords: Elderly; Starchy vegetable consumption; Cardiovascular risks; Inflammation

Posted Date: April 28th, 2020

DOI: https://doi.org/10.21203/rs.3.rs-22740/v1

License: (c) (1) This work is licensed under a Creative Commons Attribution 4.0 International License. Read Full License 


\section{Abstract \\ Objectives.}

Given the limited research on potato and other starchy vegetable consumption with cardiovascular diseases (CVD), we examined the association of potato and other starchy vegetable intakes in association with cardiovascular risk factors and inflammatory biomarkers among elderly men.

\section{Study Design.}

A cross-sectional study.

\section{Methods.}

In this study, 357 elderly men were participated. Dietary intake was assessed using food a valid and reliable frequency questionnaires. Two separated groups were considered. 1. Potato, 2. Other Starchy vegetables including corn, squash, green pea, and green lima beans. CVD risk factors including HDL, LDL, FBS, TG, TC, Alkaline phosphatase, Fibrinogen, Insulin, IL-6, TNF-a, SGOT and SGPT were measured.

\section{Results.}

Participants in the highest tertile of potato consumption had a $45 \%$ lower serum level of fasting blood sugar (OR: $0.55 ; 95 \% \mathrm{Cl}$ : 0.31, 1.97; P trend: 0.041). However, this significant association disappeared after controlling for confounders. The participants in the highest tertile of potato consumption had higher serum level of triglyceride (OR: 4.52 95\% Cl: 1.10, 18.56; P trend: 0.030 ). Moreover, participants in the highest tertiles of other starchy vegetable consumption had an $84 \%$ lower serum level of alanine aminotransferase (OR: 0.16 95\% Cl: 0.03, 0.90; P trend: 0.040).

\section{Conclusion.}

Overall, more consumption of potato may be related to developing CVD risk factors. Future research is needed to elucidate the association between potato and other starchy vegetable consumption with cardiovascular diseases risk factors in both genders.

\section{Introduction}

Cardiovascular diseases (CVDs) are the leading cause of morbidity and mortality in the American countries (1), European countries (2) and Asian countries (3). In middle eastern countries, the prevalence of CVDs has increased at an alarming rate due to changes in lifestyle and dietary pattern (4). According to the World Health Organization (WHO), CVDs have become an enormous threat to humans health, and account for $31 \%$ of all global deaths (5). In addition to high morbidity and mortality, CVDs can lead to several disabilities and compromise living standards, subsequently increasing the socioeconomic burden (68). Recent literature has demonstrated that nutrient intake and food and dietary pattern play a preventive role against coronary heart disease (CHD) (9). Although in the past few years the life expectancy of the Iranian population has improved, this has been achieved at a cost of increased age-related diseases such as CVD risk factors, abdominal obesity, insulin resistance and dyslipidemia (10).

Cardiovascular diseases are known a form of chronic non-infectious diseases related to substantial complicated risk factors such as imbalanced diet, smoking, high blood pressure, hyperlipidemia, diabetes, overweight and obesity, metabolic syndrome, excessive alcohol consumption, and a lack of physical activity $(8,11,12)$. Diet is a well-established changeable factor which can prevent and treat CVD. Moreover, after rice and wheat, the potato is the third most carbohydrate-rich food product $(13,14)$. Potato is a great source of carbohydrates, antioxidants, vitamins, and minerals. Similar to leguminous and other root vegetables, the 
potato is a good source of potassium (15). In addition to its nutritional properties, the potato may play a role in human health. It may influence on appetite, satiety property, and weight gain. However, the potato has been considered as a high glycemic index (GI) food, which may be related to the high prevalence of obesity $(16,17)$.

According to epidemiological surveys, chronic noninvasive diseases have led to increase in the Middle East. It is reported that cardiovascular disease is responsible for 25 up to $45 \%$ of deaths in these countries (18). Furthermore, CVD mortality has significantly decreased in most developed countries in recent decades while in the Middle East still rising (19). Therefore, the identification of factors related to cardiovascular risk factors in these countries is of particular importance

Potatoes are one of the most economical food crop containing several key nutrients, including potassium, vitamin $\mathrm{C}$ and dietary fiber (20). Evidence has shown that carbohydrate-rich food like potatoes and other starchy vegetables such as corn, squash, green pea, and green lima beans can regulate postprandial glucose, insulin responses and therefore can be linked to metabolic diseases like diabetes and CVDs. Also, the long term high consumption of potato has been considered as one of the important risk factors for the development of CVDs. In contrast, foods with low glycemic index foods such as whole-grain-rich diet can reduce and even prevent the risk of metabolic diseases (21).

To the best of our knowledge, there is no study about the association of potato and cardiovascular diseases among elderly subjects. Therefore, this study designed and implemented to determine the association of potato and other starchy vegetable consumption with cardiovascular diseases (CVD) risk factors in Iranian elderly.

\section{Methods}

In this cross-sectional study, the sample was collected from ten health center from south of Tehran, Iran. We selected men by cluster random sampling from these health centers (March to August 2017). We included men more than 60 years old, which were not restricted to any particular diet. Men with a malignant disease like a tumor and those who had over or under-reported total energy intake were excluded from this study ( $>4200$ or $<800 \mathrm{kcal} /$ day) (22). For estimating sample size we considered high sensitive C-reactive protein as a main dependent variable, we set $\alpha=0.05, \beta=4 \%$ and effect size $=1.5$. Based on high sensitive

C-reactive protein, the sample size was estimated to be 313. Keeping in the view of over or under-reported total energy intake, we selected 365 individual. After evaluation 8 people were excluded due to over or under-reported total energy intake and the final sample size was 357 . Ethical approval for this protocol was given by the National Institute for Medical Research Development (Grant and Ethics Number: 965430).

\section{Anthropometric assessment}

All participants were assessed for anthropometric indices including weight, height, waist circumference (WC), and body mass index (BMI). We used calibrated digital scales (SECA 831, Germany) for body weight, we asked the participants that remove their heavy clothing and shoes before recording the body weight. Height was measured while participants stood against the wall in an upright position without shoes. WC was measured over light clothing from the narrowest part of the waist with no pressure on breathing. We measured the BMI from the following formula:

$$
B M I=\frac{\text { Weight }(\mathrm{kg})}{\text { Height }\left(m^{2}\right)}
$$

\section{Dietary assessment}

Skilled nutritionist interviewed people face to face in order to obtain the usual dietary intake from 168-items semi-quantitative food frequency questionnaire (FFQ). The reliability and validity of this questionnaire have been previously tested (23). On a daily, weekly or monthly basis the participants were directed to record food consumption, the reported quantities of food consumption were converted into gram. We used the latest version of NUTRITIONIST IV for Iranian foods to calculate the average of nutrition and energy intake (24). 
To evaluate the association between dietary intake and CVD risk factors, we considered two separated groups. 1. Potato, 2. Other Starchy vegetables including corn, squash, green pea, and green lima beans.

\section{Biochemical assessment}

To measure the blood biomarkers, a blood sample was taken from each subject after 12 hours of fasting. An ultrasensitive latexenhanced immunoturbidimetric assay (Randox Laboratory Ltd., Belfast, UK) was used to measure high-sensitive C- reactive protein (hs-CRP) concentration. Triglycerides (TG), lipid profiles including total cholesterol (TC), low-density lipoprotein cholesterol (LDL-C), high-density lipoprotein cholesterol (HDL-C) and serum concentrations for fasting blood sugar (FBS). Enzyme-linked immunosorbent assay (ELISA) method (Boster Biological Technology, China) was used for determining the serum level of inflammatory biomarkers (IL-6 and TNF-a). Clauss clothing method was used to determine the rate of fibrinogen conversion into fibrin. Homeostasis Model Assessment for Insulin Resistance (HOMA-IR) (25) and Quantitative Insulin-sensitivity Check Index (QUICKI) (26) were used to assess insulin resistance and insulin sensitivity, respectively.

\section{Other variables assessments}

In this study, blood pleasure (BP) was measured when participants were sitting comfortably for at least 10 minutes. The BP was recorded twice with at least 30-second gap between them, an average of both secondaries was considered as the final BP. To assess socioeconomic status (SES), a reliable and validated questionnaire was used which was developed previously for determining the association between health outcomes and SES in Iran. The SES questionnaire included the number of family members, occupation, level of education, ownership of personal car and home, modern home appliances and the number of local and international trips throughout the last year. The SES score was calculated based on the total score for each participant. Moreover, the participants were asked about using smoking, supplements, and medication.

\section{Statistical analysis}

Kolmogorov-Smirnov test was used to determine the normal distribution of all variables. Analysis of variance (One-way ANOVA) and Chi-square tests were used for compression of quantitative and qualitative variables, respectively. Analysis of covariance (ANCOVA) was used to assess the distribution of nutrients and food groups among tertiles of potato and other starchy vegetable group which was adjusted for energy intake. ANCOVA was used to check the association between potato consumption and CVDs. Different models were used to show any significant association including model 1 which was adjusted for age, sex, SES, $\mathrm{BMI}$, and energy intake. Model 2 as a further adjusted model (model 1 plus smoking, and total dietary fiber intake). Also, model 3 which was adjusted for model 2 plus supplements consumption and medication.

Binary logistic was used to determine the odds ratio and confidence interval of CVD risk factors. Model 1 was adjusted for age, sex, SES, BMI, and energy intake while Model 2 is a further adjusted model (model 1 plus fat intake, smoking, and total dietary fiber intake). Model 3 was adjusted for model 2 plus supplements consumption and medication. SPSS version 16 used for statistical analysis and $\mathrm{P}<0.05$ considered as a significant value.

\section{Results}

Table 1 depicts that there was a significant association between age $(P=0.002)$, weight $(P=0.010)$ and WC $(P<0.0001)$ of participants with a medium and high level of potato consumption. The SES score was higher in low and high potato consumption groups $(P=0.002)$. In addition, age was greater in people who consumed lower other starchy vegetables $(P<$ 0.0001). There was a significant association between SES score and consumption of other starchy vegetables $(P<0.0001)$.

Dietary intake of participants has shown in Table 2. Participants' energy intake was more in medium and high potato consumption groups $(P<0.0001)$. Furthermore, energy intake $(P<0.0001)$ and fat intake $(P<0.0001)$ were higher in people who consumed more other starchy vegetables. Protein intake $(P=0.004)$ and calcium level $(P=0.002)$ were greater in medium and high potato consumption groups. Further, fiber intake was greater in participants who consumed more other starchy vegetables $(P=0.001)$. Moreover, participants in the higher tertile of potato consumption consumed higher amounts of vitamin $B 12(P<$ 0.0001), vitamin $A(P=0.001)$, calcium intake $(P=0.002)$. Additionally, Vitamin $B 1(P<0.0001)$, vitamin $B 6(P<0.0001)$, vitamin 
B12 $(P=0.001)$, vitamin A $(P<0.0001)$, vitamin $C(P<0.0001)$, Iron $(P<0.0001)$, vegetables $(P<0.0001)$, grain $(P<0.0001)$ intake were higher among participants who consumed more other starchy vegetables.

Biochemical markers among different levels of potato and other starchy vegetable consumption are demonstrated in Table 3. FBS level was significantly higher among those participants who consumed more other starchy vegetable as compared to participants who consumed more potato. Furthermore, TC $(P<0.0001)$ and SGOT $(P<0.0001)$ level were significantly greater in participants who consumed more potato.

Odd ratio and $95 \%$ confidence intervals of cardiovascular risk factors among participants based on the different amount of the potato and other starchy vegetable consumption illustrated in Table 4. The participants in the highest tertile of potato consumption had a $45 \%$ lower serum level of FBS (OR: $0.55 ; 95 \% \mathrm{Cl}: 0.31,1.97 ; \mathrm{P}$ trend: 0.041 ). The participants in the highest tertile of potato consumption had 4.52 time greater serum level of TG (OR: 4.52 95\% Cl: 1.10, 18.56; P trend: 0.030). Moreover, participants in the highest tertile of other starchy vegetable consumption had an $84 \%$ lower serum level of SGPT (OR: $0.1695 \% \mathrm{Cl}$ : 0.03, 0.90; $P$ trend: 0.040).

\section{Discussion}

In the current cross-sectional study, we observed a positive association between potato consumption and serum level of TG and HDL among elderly men. Furthermore, other starchy vegetable consumption was inversely associated with serum level of SGPT among elderly men.

To the best of our knowledge, this is the first study designed to determine the association between CVD risk factors and potato and other starchy vegetable consumption in elderly men. We observed no evidence that potato consumption was associated with the risk of major CVD events or mortality from CVD.

Our findings illustrated positive association with potato consumption and serum level of TG. Animal studies on rats reported reduced cholesterol and TG levels with potato diet for 3 weeks $(27,28)$. Similarly, epidemiological studies demonstrated decline in serum TG level with potato consumption (29). In the same way, our study showed the positive association of potato consumption with serum TG level.

A cross-sectional study among 4774 men and women subjects has been found a positive significant association between potato intake and insulin resistance (IR), diabetes type 2 and FBS serum level. Additionally, after adjustment to age, sex, physical activity, and dietary intake the association between FBS and diabetes type 2 with potato intake remained significant while between IR and potato intake disappeared (21). In this study, we failed to identify the relationship between potato consumption and diabetes type 2 and FBS, because the subjects we studied were only elderly men while their subjects were in both gender and the sample size for our study is smaller. Moreover comparatively our study has more adjusted viable such as age, sex, SES, BMI, fat intake, smoking, total dietary fiber intake, supplements consumption, and medication. Another cross-sectional study among 357 elderly men found a positive association between DIL (Dietary Insulin Load) and serum level of FBS. The positive significant association observed between high DIL with FBS serum levels after adjustment to energy intake, marital status, socioeconomic status, smoking, disease status, anti-diabetic drugs, thyroid drugs, and heart disease drugs (30). Mentioned studies observed a positive association between DIL and FBS. It means that the long-term consumption of high insulin index foods caused $\beta$ cell dysfunction and this situation subsequently caused IR and increased serum level of glucose (31).

This study showed a positive association of potato consumption with decrease serum level of HDL and increase serum level of TG, but we could not find an association with other lipid profiles.

We observed noticeable increase in the mean of total cholesterol in highest versus lowest tertile of potato consumption. But based on our result there was high TC in participants who had a higher consumption of potato rather than low consumption of potato. Although this increase in cholesterol has no clinical significance.

However, we observed lower LDL-C serum level in participants who consumed more other starchy vegetable while higher LDL-C serum level observed in subjects who consumed higher potato. In our study, there were few subjects with TC more than 200 and a 
high level of LDL-C for that's why we weren't able to consider the odds ratio of TC and LDL-C related to potato consumption. We found this association according to mean. The high plasma of HDL-C play a protective role for myocardial infarction and reduce coronary heart disease (32-34). The result of a cross-sectional study with 2045 among different race in both gender depicts the consumption of fried potatoes was over $75 \%$ across groups and was associated with higher odds dyslipidemia (high non-HDL cholesterol). All unhealthy foods measured were consumed more often by males as compared to females (35). Another crosssectional study $(n=12514)$ among men and women observed a positive association between potato consumption and low HDLC level (36). Phosphorus content of other starchy vegetables has other favorable properties, as it slows digestion and absorption and thus lowers blood lipid level. Its fiber content also lowers blood cholesterol level $(37,38)$.

Our result observed a high level of alkaline phosphatase (ALP) enzyme in subjects who had higher potato consumption than those participants who consumed lesser potato it seems that higher potato consumption can cause to leads higher ALP enzyme level. While lower ALP enzyme level observed among subjects who had higher other starchy vegetable consumption.

We found an inverse association between other starchy vegetable consumption and serum level of SGPT.

Moreover, a case-control study among 229 Japanese adults (men and women) with T2DM, who were not taking anti-diabetic medication showed positive correlation of total carbohydrate intake with $\mathrm{HbA1c}$. However, our study with healthy individuals has demonstrated the positive association between other starchy vegetable consumption and higher serum insulin level (39).

This is the first study which examined the association between other starchy vegetables and potato consumption and CVD risk factors among elderly individuals. Limited research is available on the association between other starchy vegetable and potatoes consumption and cardiovascular risk factors. Moreover, not all published studies have comprehensively taken into account different cardiovascular risk factors. However, in the present study, glycemic parameters, lipid profile and also inflammatory biomarkers were investigated in order to provide better insight into the association with CVD risk factors. Additionally, elderly individuals are at higher risk of cardiovascular disease and a little information is available about dietary patterns and indices in the elderly, therefore examining the association between other starchy vegetables, carbohydrate and cardiovascular risk factors is important.

The current study has several limitations that should be addressed in the interpretation of the findings. First, this study was designed as a cross-sectional study which it prevents to say causation of outcome and exposure and it is nuclear that maybe participants consumed potato for reducing CVD outcomes. Therefore, it is necessary to study the probable relationship in future prospective studies. It should be noted that the remaining effects of the unknown confounders should be considered in future studies. Another limitation of the study was that the study was conducted on elderly men, although women and men may have different dietary patterns.

\section{Conclusion}

In this cross-sectional study, potato may be related to developing CVD risk factors such as TG. However, more research is needed to illustrate the association between other starchy vegetable consumption and CVD risk factors and to understand potential differences by gender and race. Perspective studies may be needed to confirm our findings.

\section{Declarations}

\section{Ethical Approval and Consent to participate}

Ethical approval for this protocol was given by the National Institute for Medical Research Development (Grant and Ethics Number: 965430). Moreover, all included participants signed the written consent.

\section{Consent for publication}

Not Applicable.

\section{Availability of supporting data}


Not Applicable.

\section{Competing interests}

The authors declare that they have no conflict of interest.

\section{Funding}

The present study was supported by the National Institute for Medical Research Development (Grant and Ethics Number: 965430).

\section{Authors' contributions}

LA provided the idea of this study. MB, ED, and LA designed this study. LA supervised the study. MB conducted the study and ED performed the statistical analyses. MB prepared a first draft of the manuscript, and ED and LA finalized it.

\section{Acknowledgements}

We thank to all subjects who participated in this study.

\section{Authors' information}

Musral Basiry: MSc student in Public health nutritional

Elnaz Daneshzad: Ph.D.

Hadis Mozaffari: MSc. candidate in Nutritional Sciences

Leila Azadbakht: Ph.D. in Nutritional Sciences

\section{References}

1. Greenlund KJ, Croft JB, Mensah GA. Prevalence of heart disease and stroke risk factors in persons with prehypertension in the United States, 1999-2000. Arch Intern Med. 2004;164(19):2113-8.

2. Graham I, Atar D, Borch-Johnsen K, Boysen G, Burell G, Cifkova R, et al. European guidelines on cardiovascular disease prevention in clinical practice: executive summary: Fourth Joint Task Force of the European Society of Cardiology and Other Societies on Cardiovascular Disease Prevention in Clinical Practice (Constituted by representatives of nine societies and by invited experts). Eur Heart J. 2007;28(19):2375-414.

3. Azizi F, Rahmani M, Emami H, Mirmiran P, Hajipour R, Madjid M, et al. Cardiovascular risk factors in an Iranian urban population: Tehran lipid and glucose study (phase 1). Soz Praventivmed. 2002;47(6):408-26.

4. Ebrahimi M, Kazemi-Bajestani SM, Ghayour-Mobarhan M, Ferns GA. Coronary artery disease and its risk factors status in iran: a review. Iran Red Crescent Med J. 2011;13(9):610-23.

5. Organization WH. Hearts: technical package for cardiovascular disease management in primary health care (WHO, 2016). Google Scholar.

6. Yazdanyar A, Newman AB. The burden of cardiovascular disease in the elderly: morbidity, mortality, and costs. Clin Geriatr Med. 2009;25(4):563-77. vii.

7. Zaina S, Lund G. Epigenetics: a tool to understand diet-related cardiovascular risk? J Nutrigenet Nutrigenomics. 2011;4(5):261-74.

8. Praveen PA, Roy A, Prabhakaran D. Cardiovascular disease risk factors: a childhood perspective. Indian J Pediatr. 2013;80(Suppl 1):3-12.

9. Azadbakht L, Esmaillzadeh A. Dietary and non-dietary determinants of central adiposity among Tehrani women. Public Health Nutrition. 2007;11(5):528-34. 
10. Sarrafzadegan N, Gharipour M, Sadeghi M, Khosravi AR, Tavassoli AA. Metabolic syndrome in Iranian elderly. ARYA Atheroscler. 2012;7(4):157-61.

11. Walker J. Reducing cardiovascular disease risk: cholesterol and diet. Nurs Stand. 2013;28(2):48-55.

12. Gómez de la Cámara A, Ángeles Gómez Mateos M, Ferrando Vivas P, Teresa Barianca Oyagüe M, Abiatua Borda I. Posada de la Paz M. Prevalencia de factores de riesgo cardiovascular en una cohorte de afectados por el síndrome del aceite tóxico. Medicina Clínica. 2003;121(11):405-7.

13. Brand-Miller JC. Postprandial glycemia, glycemic index, and the prevention of type 2 diabetes. Oxford University Press; 2004.

14. Council BP. Target market report for the export of GB seed potatoes. BPC Target Market Report Russia. 2006.

15. Wolk A, Larsson SC. Potato consumption and risk of cardiovascular disease: 2 prospective cohort studies. The American Journal of Clinical Nutrition. 2016;104(5):1245-52.

16. McGill CR, Kurilich AC, Davignon J. The role of potatoes and potato components in cardiometabolic health: A review. Ann Med. 2013;45(7):467-73.

17. Geliebter A, Lee MIC, Abdillahi M, Jones J. Satiety following Intake of Potatoes and Other Carbohydrate Test Meals. Annals of Nutrition Metabolism. 2013;62(1):37-43.

18. Yusuf S, Reddy S, Ounpuu S, Anand S. Global burden of cardiovascular diseases: Part II: variations in cardiovascular disease by specific ethnic groups and geographic regions and prevention strategies. Circulation. 2001;104(23):2855-64.

19. Yusuf S, Hawken S, Ounpuu S, Dans T, Avezum A, Lanas F, et al. Effect of potentially modifiable risk factors associated with myocardial infarction in 52 countries. 2004:937-52.

20. Drewnowski A. The Nutrient Rich Foods Index helps to identify healthy, affordable foods. Am J Clin Nutr. 2010;91(4):1095S$101 \mathrm{~S}$.

21. Khosravi-Boroujeni H, Mohammadifard N, Sarrafzadegan N, Sajjadi F, Maghroun M, Khosravi A, et al. Potato consumption and cardiovascular disease risk factors among Iranian population. Int J Food Sci Nutr. 2012;63(8):913-20.

22. Saraf-Bank S, Haghighatdoost F, Esmaillzadeh A, Larijani B, Azadbakht L. Adherence to Healthy Eating Index-2010 is inversely associated with metabolic syndrome and its features among Iranian adult women. Eur J Clin Nutr. 2017;71(3):42530 .

23. Mirmiran P, Esfahani FH, Mehrabi Y, Hedayati M, Azizi F. Reliability and relative validity of an FFQ for nutrients in the Tehran lipid and glucose study. Public Health Nutr. 2010;13(5):654-62.

24. Mozaffari H, Namazi N, Larijani B, Surkan PJ, Azadbakht L. Associations between dietary insulin load with cardiovascular risk factors and inflammatory parameters in elderly men: a cross-sectional study. Br J Nutr. 2019:1-19.

25. Li S, Yin C, Zhao W, Zhu H, Xu D, Xu Q, et al. Homeostasis model assessment of insulin resistance in relation to the poor functional outcomes in nondiabetic patients with ischemic stroke. Biosci Rep. 2018;38(3).

26. Katz A, Nambi SS, Mather K, Baron AD, Follmann DA, Sullivan G, et al. Quantitative insulin sensitivity check index: a simple, accurate method for assessing insulin sensitivity in humans. J Clin Endocrinol Metab. 2000;85(7):2402-10.

27. Robert L, Narcy A, Rayssiguier Y, Mazur A, Remesy C. Lipid metabolism and antioxidant status in sucrose vs. potato-fed rats. J Am Coll Nutr. 2008;27(1):109-16.

28. Robert L, Narcy A, Rock E, Demigne C, Mazur A, Rémésy C. Entire potato consumption improves lipid metabolism and antioxidant status in cholesterol-fed rat. European Journal of Nutrition \$V. 2006;45(5):267-74.

29. Ness AR, Powles JW. Fruit and vegetables, and cardiovascular disease: a review. Int J Epidemiol. 1997;26(1):1-13.

30. Mozaffari H, Namazi N, Larijani B, Surkan PJ, Azadbakht L. Associations between dietary insulin load with cardiovascular risk factors and inflammatory parameters in elderly men: a cross-sectional study. Br J Nutr. 2019;121(7):773-81.

31. Nimptsch K, Brand-Miller JC, Franz M, Sampson L, Willett WC, Giovannucci E. Dietary insulin index and insulin load in relation to biomarkers of glycemic control, plasma lipids, and inflammation markers. Am J Clin Nutr. 2011;94(1):182-90.

32. Hewing B, Moore KJ, Fisher EA. HDL and cardiovascular risk: time to call the plumber? Circ Res. 2012;111(9):1117-20.

33. Navab M, Reddy ST, Van Lenten BJ, Fogelman AM. HDL and cardiovascular disease: atherogenic and atheroprotective mechanisms. Nature Reviews Cardiology. 2011;8:222. 
34. Santos-Gallego CG, Torres F, Badimón JJ. The beneficial effects of HDL-C on atherosclerosis: rationale and clinical results. Clinical Lipidology. 2011;6(2):181-208.

35. Vaccaro JA, Zarini GG, Huffman FG. Cross-sectional analysis of unhealthy foods, race/ethnicity, sex and cardiometabolic risk factors in U.S. adults. Nutr Diet. 2018;75(5):474-80.

36. Khosravi-Boroujeni H, Mohammadifard N, Sarrafzadegan N, Sajjadi F, Maghroun M, Khosravi A, et al. Potato consumption and cardiovascular disease risk factors among Iranian population. Int J Food Sci Nutr. 2012;63(8):913-20.

37. Azadbakht L, Surkan PJ, Esmaillzadeh A, Willett WC. The Dietary Approaches to Stop Hypertension eating plan affects Creactive protein, coagulation abnormalities, and hepatic function tests among type 2 diabetic patients. J Nutr. 2011;141(6):1083-8.

38. Lazarov KaW MJ. Hypocholesterolemic effect of potato peels as a dietary fibre source. Med Sci Res. 1996.

39. Haimoto H, Watanabe S, Komeda M, Wakai K. The impact of carbohydrate intake and its sources on hemoglobin A1c levels in Japanese patients with type 2 diabetes not taking anti-diabetic medication. Diabetes Metab Syndr Obes. 2018;11:53-64.

\section{Tables}

phic characteristics of participants in different tertiles of the potato and other starchy vegetable consumption $(\mathrm{N}=357)$

\begin{tabular}{|c|c|c|c|c|c|c|c|c|c|}
\hline & $\begin{array}{c}\text { All } \\
(n=357)\end{array}$ & \multicolumn{3}{|c|}{ Tertiles of potato consumption } & P-value * & \multicolumn{3}{|c|}{ Tertiles of other Starchy vegetable consumption } & P-value* \\
\hline & $64.96 \pm 6.51^{\mathrm{a}}$ & $63.3 \pm 7.4$ & $65.8 \pm 6.2$ & $65.8 \pm 5.3$ & 0.002 & $66.5 \pm 7.2$ & $65.5 \pm 7.5$ & $62.9 \pm 3.3$ & $<0.0001$ \\
\hline & $72.18 \pm 10.24$ & $70.4 \pm 10.3$ & $74.3 \pm 9.3$ & $71.7 \pm 10.8$ & 0.010 & $73.7 \pm 7.3$ & $69.3 \pm 12.3$ & $72.9 \pm 10.9$ & 0.002 \\
\hline & $25.35 \pm 3.19$ & $24.6 \pm 2.9$ & $26.0 \pm 3.5$ & $25.5 \pm 2.8$ & 0.001 & $26.0 \pm 3.0$ & $24.7 \pm 3.3$ & $25.0 \pm 3.1$ & 0.002 \\
\hline & $13.03 \pm 1.62$ & $13.4 \pm 1.7$ & $12.6 \pm 1.3$ & $13.0 \pm 1.8$ & 0.002 & $12.8 \pm 1.5$ & $12.7 \pm 1.1$ & $13.6 \pm 1.9$ & $<0.0001$ \\
\hline & $0.173 \pm 0.37$ & & & & & & & & \\
\hline & & $23(37.1)$ & $25(40.3)$ & $14(22.6)$ & 0.302 & $15(24.2)$ & $23(37.1)$ & $24(38.7)$ & 0.011 \\
\hline \multirow{5}{*}{ 6) } & & $3(17.6 \%)$ & $9(52.9 \%)$ & $5(29.4 \%)$ & 0.191 & $5(29.4 \%)$ & $10(58.8 \%)$ & $2(11.8 \%)$ & 0.015 \\
\hline & & $122(39.9 \%)$ & $114(33.5 \%)$ & $104(30.6 \%)$ & 0.191 & $42(41.8 \%)$ & $92(27.1 \%)$ & $106(31.2 \%)$ & 0.015 \\
\hline & $0.518 \pm 0.50$ & & & & & & & & \\
\hline & & $74(40.0 \%)$ & $55(29.7 \%)$ & $56(30.3 \%)$ & 0.073 & $86(46.5 \%)$ & $59(31.9 \%)$ & $40(21.6 \%)$ & 0.001 \\
\hline & & $51(29.7 \%)$ & 68 (39.5\%) & $53(30.8 \%)$ & 0.073 & $61(35.5 \%)$ & $43(25.0 \%)$ & $68(39.5 \%)$ & 0.001 \\
\hline
\end{tabular}

ndex; WC, Waist Circumference, SES, socioeconomic status

lted from ANOVA

d deviation 
2. Dietary intakes of participants in different tertiles of the potato and other starchy vegetable consumption $(n=357)$

\begin{tabular}{|c|c|c|c|c|c|c|c|c|}
\hline \multirow[b]{2}{*}{ Jles } & \multicolumn{3}{|c|}{ Tertiles of potato consumption } & \multirow[b]{2}{*}{ P-value* } & \multicolumn{3}{|c|}{ Tertiles of other Starchy vegetable consumption } & \multirow[b]{2}{*}{ p-value* } \\
\hline & $\begin{array}{c}\text { Low } \\
(n=125)\end{array}$ & $\begin{array}{l}\text { Medium } \\
(n=123)\end{array}$ & $\begin{array}{c}\text { High } \\
(n=109)\end{array}$ & & $\begin{array}{c}\text { Low } \\
(n=147)\end{array}$ & $\begin{array}{l}\text { Medium } \\
(n=102)\end{array}$ & $\begin{array}{c}\text { High } \\
(n=108)\end{array}$ & \\
\hline ry (Kcal/d) & $1908.3 \pm 29.1^{\mathrm{a}}$ & $2282.2 \pm 49.8$ & $2293.0 \pm 60.9$ & $<0.0001$ & $1981.8 \pm 45.2$ & $2352.2 \pm 54.1$ & $2288.9 \pm 60.0$ & $<0.0001$ \\
\hline $\mathrm{n}(\mathrm{g} / \mathrm{d})$ & $86.7 \pm 1.8$ & $84.4 \pm 1.8$ & $77.9 \pm 1.9$ & 0.004 & $82.71 \pm 1.7$ & $84.69 \pm 2.0$ & $82.66 \pm 1.9$ & 0.709 \\
\hline r/d) & $60.6 \pm 1.5$ & $61.9 \pm 1.5$ & $60.1 \pm 1.6$ & 0.660 & $56.47 \pm 1.3$ & $63.32 \pm 1.6$ & $61.09 \pm 1.5$ & $<0.0001$ \\
\hline hydrate $(\mathrm{g} / \mathrm{d})$ & $329.5 \pm 4.1$ & $336.9 \pm 4.0$ & $342.8 \pm 4.3$ & 0.086 & $342.3 \pm 3.7$ & $322.8 \pm 4.4$ & $340.2 \pm 4.2$ & 0.002 \\
\hline $\mathrm{mg} / \mathrm{d})$ & $17.2 \pm 0.57$ & $16.9 \pm 0.56$ & $16.5 \pm 0.60$ & 0.685 & $16.94 \pm 1.7$ & $19.31 \pm 0.6$ & $14.40 \pm 0.6$ & 0.305 \\
\hline$I(\mathrm{mg} / \mathrm{d})$ & $18.85 \pm 0.60$ & $17.96 \pm 0.59$ & $17.19 \pm 0.63$ & 0.177 & $16.79 \pm 0.5$ & $20.05 \pm 0.64$ & $17.83 \pm 0.62$ & 0.001 \\
\hline$(\mathrm{mg} / \mathrm{d})$ & $13.17 \pm 0.35$ & $12.78 \pm 0.34$ & $12.58 \pm 0.36$ & 0.502 & $12.47 \pm 0.3$ & $14.33 \pm 0.4$ & $11.98 \pm 0.5$ & $<0.0001$ \\
\hline in B1 (mg/d) & $1.560 \pm 0.03$ & $1.517 \pm 0.03$ & $1.496 \pm 0.03$ & 0.437 & $1.44 \pm 0.03$ & $1.47 \pm 0.03$ & $1.69 \pm 0.03$ & $<0.0001$ \\
\hline in B6 (mg/d) & $1.777 \pm 0.04$ & $1.929 \pm 0.04$ & $1.888 \pm 0.04$ & 0.039 & $1.64 \pm 0.03$ & $1.91 \pm 0.04$ & $2.11 \pm 0.04$ & $<0.0001$ \\
\hline in B12 $(\mu \mathrm{g} / \mathrm{d})$ & $8.687 \pm 1.35$ & $16.44 \pm 1.33$ & $11.16 \pm 1.41$ & $<0.0001$ & $8.62 \pm 1.25$ & $16.0 \pm 1.47$ & $13.19 \pm 1.42$ & 0.001 \\
\hline in $C(m g / d)$ & $209.6 \pm 8.00$ & $234.6 \pm 6.88$ & $217.5 \pm 4.23$ & 0.082 & $200.4 \pm 7.26$ & $250.4 \pm 8.56$ & $220.1 \pm 8.25$ & $<0.0001$ \\
\hline in $A(R A E / d)$ & $1226 \pm 48.9$ & $1452 \pm 46.5$ & $1462 \pm 50.4$ & 0.001 & $1210 \pm 44.0$ & $1402 \pm 51.9$ & $1485 \pm 50.1$ & $<0.0001$ \\
\hline esium (mg/d) & $329.6 \pm 6.99$ & $320.5 \pm 6.89$ & $208.6 \pm 7.32$ & 0.125 & $322.0 \pm 6.50$ & $315.0 \pm 7.67$ & $322.1 \pm 7.39$ & 0.641 \\
\hline Im $(\mathrm{mg} / \mathrm{d})$ & $1621.2 \pm 60.7$ & $1508.5 \pm 59.8$ & $1374.3 \pm 63.5$ & 0.002 & $1569.6 \pm 56.3$ & $1531.7 \pm 66.5$ & $1398.4 \pm 64.1$ & 0.124 \\
\hline $\operatorname{sium}(\mathrm{mg} / \mathrm{d})$ & $4240.7 \pm 89.7$ & $4001.6 \pm 88.4$ & $3954.2 \pm 93.9$ & 0.066 & $3993 \pm 83.4$ & $4139 \pm 98.4$ & $4113 \pm 94.8$ & 0.486 \\
\hline $\mathrm{mg} / \mathrm{d})$ & $9.401 \pm 0.31$ & $9.553 \pm 0.30$ & $8.670 \pm 0.32$ & 0.112 & $9.66 \pm 0.28$ & $9.01 \pm 0.34$ & $9.83 \pm 0.32$ & 0.142 \\
\hline able (g/d) & $382.8 \pm 15.3$ & $413.5 \pm 15.0$ & $364.5 \pm 16.0$ & 0.075 & $351.8 \pm 13.9$ & $441.7 \pm 16.4$ & $386.0 \pm 14.8$ & $<0.0001$ \\
\hline Irocessed meat & $9.482 \pm 0.54$ & $8.385 \pm 0.54$ & $11.05 \pm 0.57$ & 0.003 & $9.826 \pm 0.51$ & $10.30 \pm 0.60$ & $8.574 \pm 0.58$ & 0.095 \\
\hline Ind chicken $(g / d)$ & $49.01 \pm 2.28$ & $49.23 \pm 2.25$ & $40.30 \pm 2.39$ & 0.009 & $41.53 \pm 2.11$ & $52.41 \pm 2.48$ & $47.55 \pm 2.39$ & 0.005 \\
\hline$(\mathrm{g} / \mathrm{d})$ & $358.3 \pm 13.4$ & $297.6 \pm 13.3$ & $294.7 \pm 14.1$ & 0.010 & $383.8 \pm 11.7$ & $251.6 \pm 13.8$ & $279.6 \pm 13.3$ & $<0.0001$ \\
\hline products $(g / d)$ & $716.0 \pm 30.0$ & $585.3 \pm 29.5$ & $559.1 \pm 01.4$ & 0.001 & $643.0 \pm 28.1$ & $660.6 \pm 33.1$ & $560.5 \pm 31.5$ & 0.594 \\
\hline
\end{tabular}

eviations: PUFA, poly-unsaturated fatty acids; MUFA, monounsaturated fatty acids; SFA, saturated fatty acids.

ysis of covariance (ANCOVA; adjusted for energy intake) was used to determine P for all variables except for energy intake.

$\mathrm{n} \pm$ Standard errors

cal tests among participants based on the different tertiles of the potato and other starchy vegetable consumption $(\mathrm{N}=357)$

\begin{tabular}{|c|c|c|c|c|c|c|c|c|}
\hline \multirow[b]{2}{*}{$\begin{array}{l}\text { All }(\mathrm{N}=375) \\
\quad(\mathrm{m} \pm \mathrm{SD})\end{array}$} & \multicolumn{3}{|c|}{ Tertiles of potato consumption } & \multirow[b]{2}{*}{ p-value* } & \multicolumn{3}{|c|}{ Tertiles of other Starchy vegetable consumption } & \multirow[b]{2}{*}{ p-value* } \\
\hline & $\begin{array}{c}\text { Low } \\
(n=125)\end{array}$ & $\begin{array}{l}\text { Medium } \\
(n=123)\end{array}$ & $\begin{array}{c}\text { High } \\
(n=109)\end{array}$ & & $\begin{array}{c}\text { Low } \\
(n=125)\end{array}$ & $\begin{array}{l}\text { Medium } \\
(n=123)\end{array}$ & $\begin{array}{c}\text { High } \\
(n=109)\end{array}$ & \\
\hline \multicolumn{9}{|l|}{$101.7 \pm 0.91^{\mathrm{a}}$} \\
\hline & $103.2 \pm 1.7$ & $100.2 \pm 1.5$ & $101.6 \pm 1.4$ & 0.386 & $106.2 \pm 1.4$ & $96.29 \pm 1.7$ & $100.0 \pm 1.5$ & $<0.0001$ \\
\hline & $102.1 \pm 1.51$ & $101.1 \pm 1.46$ & $102.0 \pm 1.43$ & 0.888 & $105.3 \pm 1.37$ & $98.12 \pm 1.59$ & $100.4 \pm 1.57$ & 0.003 \\
\hline & $102.5 \pm 1.74$ & $100.8 \pm 1.43$ & $101.9 \pm 1.48$ & 0.710 & $105.7 \pm 1.37$ & $97.99 \pm 1.58$ & $99.95 \pm 1.54$ & 0.001 \\
\hline & $102.7 \pm 1.47$ & $100.7 \pm 1.44$ & $101.8 \pm 1.49$ & 0.657 & $105.9 \pm 1.36$ & $97.49 \pm 1.59$ & $100.1 \pm 1.54$ & $<0.0001$ \\
\hline \multicolumn{9}{|l|}{$176.8 \pm 1.33$} \\
\hline & $169.1 \pm 2.3$ & $186.9 \pm 1.5$ & $174.3 \pm 2.7$ & $<0.0001$ & $177.4 \pm 2.0$ & $175.0 \pm 0.9$ & $177.8 \pm 2.2$ & 0.680 \\
\hline & $169.3 \pm 2.2$ & $186.4 \pm 2.1$ & $174.3 \pm 2.2$ & $<0.0001$ & $179.0 \pm 2.12$ & $174.3 \pm 2,48$ & $176.1 \pm 2.43$ & 0.372 \\
\hline & $169.7 \pm 2.23$ & $186.1 \pm 2.17$ & $174.3 \pm 2.20$ & $<0.0001$ & $179.7 \pm 2.20$ & $174.3 \pm 2.45$ & $175.0 \pm 2.46$ & 0.251 \\
\hline
\end{tabular}

Page $10 / 12$ 


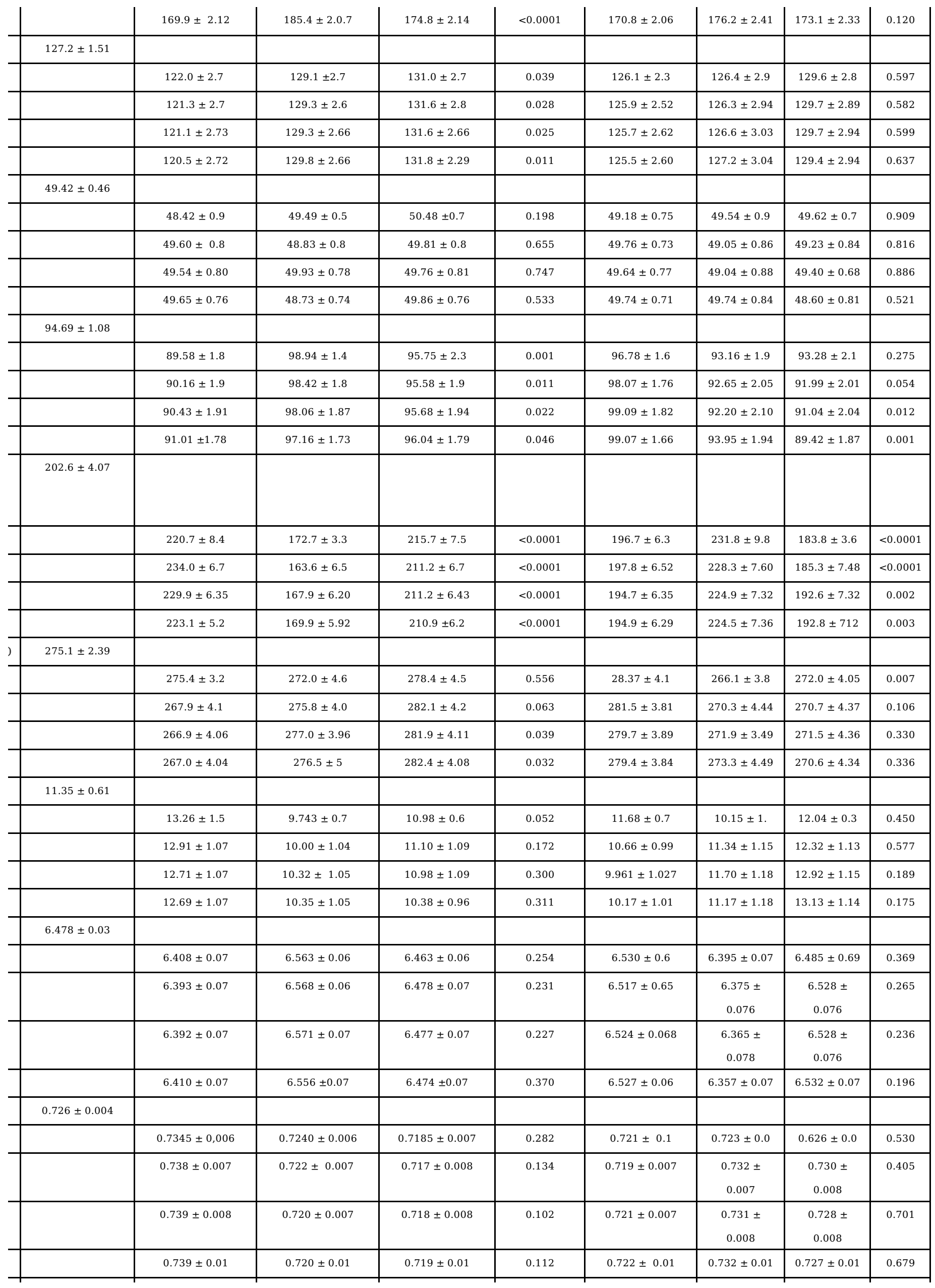




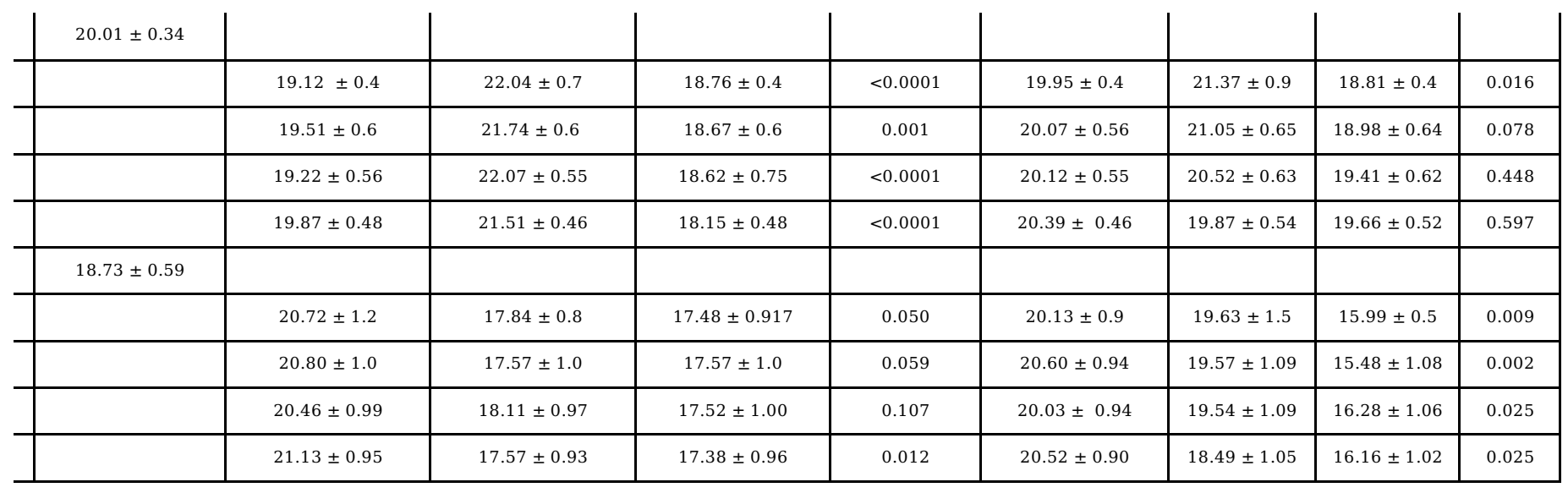

S: fasting blood sugar; TG: triglyceride; HDL \& LDL: high and low-density lipoprotein; TC: total cholesterol; AST: aspartate trance amines;.

is mean $\pm \mathrm{SE}$.

d resulted from ANCOVA analysis and in model 1 which adjusted for sex, age, SES, BMI and energy intake and model 2 which adjusted for model $1+$ smoking, and total dietary fiber 3 which adjusted for model $2+$ supplements consumption and medication.

.0 and $95 \%$ confidence intervals of cardiovascular risk factors among participants based on the different amount of the potato and other starchy vegetable consumption $(\mathrm{N}=$

\begin{tabular}{|c|c|c|c|c|c|c|c|c|}
\hline \multirow[b]{2}{*}{ iables } & \multicolumn{3}{|c|}{ Tertiles of potato consumption } & \multirow[b]{2}{*}{ P trend** } & \multicolumn{3}{|c|}{ Tertiles of other Starchy vegetable consumption } & \multirow[b]{2}{*}{ P trend** } \\
\hline & $\begin{array}{c}\text { Low } \\
(n=125)\end{array}$ & $\begin{array}{l}\text { Medium } \\
(n=123)\end{array}$ & $\begin{array}{c}\text { High } \\
(n=109)\end{array}$ & & $\begin{array}{c}\text { Low } \\
(n=125)\end{array}$ & $\begin{array}{l}\text { Medium } \\
(n=123)\end{array}$ & $\begin{array}{c}\text { High } \\
(n=109)\end{array}$ & \\
\hline \multicolumn{9}{|c|}{ l) $\mathrm{N}=118$} \\
\hline & 1 & $0.83(0.49,1.39)$ & $0.55(0.31,0.97)$ & 0.041 & 1 & $0.41(0.23,0.72)$ & $0.77(0.46,1.30)$ & 0.222 \\
\hline & 1 & $1.08(0.58,2.01)$ & $0.65(0.34,1.24)$ & 0.176 & 1 & $0.75(0.38,1.47)$ & $1.28(0.67,2.44)$ & 0.470 \\
\hline & 1 & $1.02(0.52,2.03)$ & $0.64(0.32,1.27)$ & 0.176 & 1 & $0.68(0.33,1.38)$ & $1.03(0.52,2.06)$ & 0.940 \\
\hline & 1 & $0.97(0.48,1.98)$ & $0.62(0.31,1.25)$ & 0.149 & 1 & $0.63(0.30,1.30)$ & $1.08(0.53,2.19)$ & 0.860 \\
\hline \multicolumn{9}{|c|}{$(\mathrm{N}=23)$} \\
\hline & 1 & $2.82(0.73,10.92)$ & $5.03(1.38,18.33)$ & 0.010 & 1 & $1.28(0.44,3.64)$ & $1.39(0.50,3.82)$ & 0.519 \\
\hline & 1 & $2.18(0.53,8.90)$ & $3.85(1.01,14.80)$ & 0.037 & 1 & $0.81(0.26,2.50)$ & $1.04(0.33,3.19)$ & 0.948 \\
\hline & 1 & $2.17(0.50,9.29)$ & $4.31(1.07,17.36)$ & 0.026 & 1 & $0.62(0.17,2.62)$ & $0.85(0.26,2.77)$ & 0.854 \\
\hline & 1 & $2.65(0.60,11.78)$ & $4.52(1.10,18.56)$ & 0.030 & 1 & $0.58(0.16,2.15)$ & $0.91(0.28,3.01)$ & 0.941 \\
\hline \multicolumn{9}{|c|}{.) $(N=53)$} \\
\hline & 1 & $0.48(0.22,1.05)$ & $1.18(0.60,2.30)$ & 0.662 & 1 & $0.39(0.17,0.87)$ & $0.65(0.33,1.29)$ & 0.147 \\
\hline & 1 & $0.72(0.29,1.79)$ & $1.90(0.84,4.30)$ & 0.111 & 1 & $0.35(0.13,0.90)$ & $0.77(0.34,1.74)$ & 0.497 \\
\hline & 1 & $0.78(0.30,2.04)$ & $2.67(1.12,6.38)$ & 0.025 & 1 & $0.47(0.17,1.24)$ & $1.02(0.43,2.39)$ & 0.962 \\
\hline & 1 & $0.83(0.30,2.29)$ & $3.02(1.21,7.53)$ & 0.016 & 1 & $0.38(0.13,1.10)$ & $1.78(0.68,4.36)$ & 0.306 \\
\hline \multicolumn{9}{|c|}{$. \mathrm{IU} / \mathrm{ml}) \mathrm{N}=50$} \\
\hline & 1 & $1.09(0.51,2.32)$ & $1.54(0.74,3.21)$ & 0.240 & 1 & $0.48(0.20,1.13)$ & $1.29(0.66,2.50)$ & 0.529 \\
\hline & 1 & $1.22(0.51,2.91)$ & $1.82(0.79,4.16)$ & 0.140 & 1 & $0.87(0.32,2.35)$ & $3.32(1.34,8.23)$ & 0.010 \\
\hline & 1 & $1.42(0.59,3.41)$ & $1.83(0.79,4.24)$ & 0.156 & & $1.07(0.39,2.94)$ & $5.67(2.11,15.23)$ & 0.001 \\
\hline & 1 & $1.29(0.52,3.23)$ & $1.70(0.70,4.11)$ & 0.228 & 1 & $1.01(0.35,2.89)$ & $6.15(2.25,16.77)$ & 0.001 \\
\hline \multicolumn{9}{|c|}{$(\mathrm{N}=31)$} \\
\hline & 1 & $0.84(0.36,1.96)$ & $0.59(0.22,1.54)$ & 0.286 & 1 & $0.86(0.39,1.92)$ & $0.13(0.03,0.59)$ & 0.007 \\
\hline & 1 & $0.58(0.21,1.54)$ & $0.51(0.16,1.58)$ & 0.229 & 1 & $0.95(0.37,2.43)$ & $0.14(0.02,0.68)$ & 0.016 \\
\hline & 1 & $0.57(0.20,1.58)$ & $0.57(0.18,1.75)$ & 0.308 & 1 & $1.01(0.33,3.05)$ & $0.14(0.02,0.73)$ & 0.019 \\
\hline & 1 & $0.47(0.15,1.45)$ & $0.49(0.15,1.60)$ & 0.232 & 1 & $0.80(0.24,2.60)$ & $0.16(0.03,0.90)$ & 0.040 \\
\hline
\end{tabular}

BS: fasting blood sugar; TG: triglyceride; HDL: high-density lipoprotein; SGPT: Serum glutamic pyruvic transaminase.

as odds ratio and confidence intervals; **P trends are resulted from binary logistic regression.

ted for sex, age, SES, BMI, and energy intake

ted for model $1+$ smoking, and total dietary fiber intake; Model 3 is adjusted for model $2+$ supplement consumption and medication. 